\title{
LEFT VENTRICULAR FUNCTION IN COPD PATIENTS- ITS CLINICAL AND ECHOCARDIOGRAPHIC EVALUATION
}

\author{
Saroj Mandal1 ${ }^{1}$, Souranshu Chatterjee ${ }^{2}$, Debasmita Mandal ${ }^{3}$, Ritabrata Mitra ${ }^{4}$, S. C. Mandal ${ }^{5}$ \\ ${ }_{1}^{1}$ Assistant Professor, Department of Cardiology, IPGMER and SSKM Hospital, Kolkata, West Bengal. \\ ${ }^{2} \mathrm{HOD}$, Department of Cardiology, NBMC and H, West Bengal. \\ ${ }^{3}$ Associate Professor, Department of Obstetrics and Gynaecology, ESI PGIMSR, ESIC Medical College, Joka, West Bengal. \\ ${ }^{4}$ Assistant Professor, Department of Chest Medicine, IPGMER and SSKM Hospital, Kolkata, West Bengal. \\ 5 Professor and HOD, Department of Cardiology, IPGMER and SSKM Hospital, Kolkata, West Bengal.
}

\section{ABSTRACT}

\section{BACKGROUND}

Chronic Obstructive Pulmonary Disease (COPD) is an important and rapidly increasing problem in all over the world. Right ventricular involvement in the form of pulmonary arterial hypertension and cor pulmonale occurs in COPD patients and eventually left ventricular function may get affected among such patients.

The objective of this study is to assess the left ventricular function in COPD patients with or without cor pulmonale clinically and echocardiographically.

\section{MATERIALS AND METHODS}

52 cases of COPD were included in this study for assessment of left ventricular functions by clinical and echocardiographic method. They were divided into two groups. In Group A, 42 cases of COPD without cor pulmonale were included and in Group B 10 patients of COPD with cor pulmonale were included.

\section{RESULTS}

10 COPD patients had cor pulmonale. Cor pulmonale group patients had more Electrocardiogram abnormality than non-cor pulmonale group. LV diastolic function significantly differs in two groups of patients of COPD with or without cor pulmonale. All of the $10(100 \%)$ patients with cor pulmonale and $13(31 \%)$ of the 42 patients without cor pulmonale had reduced E/A is measured by the pulse wave Doppler and e'/a' is measured by Doppler tissue imaging.

\section{CONCLUSION}

Non-invasive evaluation of left ventricular function by Echocardiography was found to be helpful in detecting left ventricular dysfunction in patient of COPD with or without cor pulmonale. It can be concluded that systolic and diastolic dysfunction can be present without being overt clinical manifestation of left ventricular dysfunction and DTI is very helpful in this regard.

\section{KEYWORDS}

Left Ventricular Function in COPD, Echo Evaluation in COPD, Cor Pulmonale and LV Function.

HOW TO CITE THIS ARTICLE: Mandal S, Chatterjee S, Mandal D, et al. Left ventricular function in COPD patients- its clinical and echocardiographic evaluation. J. Evolution Med. Dent. Sci. 2017;6(31):2512-2517, DOI: 10.14260/Jemds/2017/544

\section{BACKGROUND}

Cardiovascular disease is one of the most important cause of mortality and morbidity in COPD patients and causing hospitalisation in $50 \%$ of patients and death in $20 \%$ of patients. ${ }^{1}$ The anatomical and functional relationship between the heart and the respiratory system is so close that dysfunction of one of these systems can influence the function of the other. ${ }^{2}$ Barberà and Blanco found Pulmonary arterial hypertension (characterised haemodynamically by a resting mean pulmonary artery pressure -Ppa $>20 \mathrm{mmHg}$ ) and cor pulmonale is an important complication in the natural history of Chronic Obstructive Pulmonary Disease (COPD) and its prevalence is quite high in patients with advanced COPD. ${ }^{3}$

Financial or Other, Competing Interest: None.

Submission 14-03-2017, Peer Review 09-04-2017,

Acceptance 12-04-2017, Published 17-04-2017.

Corresponding Author:

Dr. Saroj Mandal,

Flat $5 d / 16$, Tower Olive,

Active Acres, 54/10, D. C. Dey Road,

Kolkata-700015.

E-mail: drsarojkumarr@gmail.com

DOI: $10.14260 /$ jemds $/ 2017 / 544$

\section{(c) (i) $\$$}

Evidence of cor pulmonale was found in $40 \%$ of patients with COPD in one autopsy study.[4]

In patients with severe COPD and cor pulmonale right ventricle overload with $\mathrm{RV}$ dilation occur which eventually results in abnormal LV performance. This abnormal LV performance in persons with COPD may be due to a number of factors, such as hypoxaemia and acidosis which itself directly can impair LV relaxation, ventricular interdependence (because the RV and LV share a common septum, RV dilatation may lead to bulging of the septum into the LV, which would in turn increase LV end-diastolic pressure, decrease venous return and diminish LV stroke volume and $\mathrm{CO}$ ), increased volume in lower lobes of the lung can hinder LV filling and lastly concurrent coronary artery disease; also due to more direct metabolic, haemodynamic or infectious effects upon the myocardium. Indeed LV hypertrophy accompanies RV hypertrophy in approximately $30 \%$ of patients with COPD and severe left ventricular failure, occasionally presenting as the dominant clinical feature. Therefore, cor pulmonale may be regarded as a generalised cardiomyopathy and with associated reductions in left ventricular filling, left ventricular stroke volume and cardiac output, although left ventricular ejection fraction is generally preserved. 5 


\section{Aim}

To assess the left ventricular function in COPD patients with or without cor pulmonale by echocardiography.

The criteria for selection of cases in the study were clinical or laboratory data indicating the diagnosis of COPD.

\section{Diagnostic Criteria}

i) Cough with expectoration on most of the days for 3 successive months in two consecutive years. ii). Episodic breathlessness with wheezing simulating asthma. iii) Physical examination of patient and abnormal radiological finding in chest suggestive of COPD, excluding other specific disease, iv). Pulmonary functional abnormality. study.

All patients had given written consent to take part in the

\section{Exclusion Criteria}

Patients with history or clinical evidence of hypertension, coronary heart disease, structural diseases of the heart (Valvular heart disease, congenital heart disease and cardiomyopathy), alcoholism or other recognised cause of left ventricular dysfunction and unstable cardiorespiratory status, defined as the occurrence of respiratory failure bronchopulmonary infection or congestive heart failure in the previous 2 months, also lung abscess, bronchogenic carcinoma were excluded from the study.

\section{MATERIALS AND METHODS}

The present study was carried out in Hospital and IPGMER Kolkata involving 52 patients of COPD admitted to the Department of General Medicine, Cardiology and Chest during the period from January 2015 to December 2016. Selected cases of COPD with or without cor pulmonale were studied for left ventricular dysfunction.

\section{Methods}

On admission, a detailed account of the history and physical examination was done and recorded in the proforma. Routine and laboratory investigation including determination of $\mathrm{Hb} \%$ and Total RBC count, TWBC, DC, fasting and post prandial blood sugar, Serum Urea, Serum Creatinine, Sputum Gram stain and AFB staining, culture and sensitivity, urine routine and microscopic examination was done. Also 12-lead Electrocardiogram and Chest x-ray (PA view) and pulmonary function test was done.

Patients satisfying the diagnostic criteria of COPD were included in the present study, out of which 42 patients of COPD without evidence of cor pulmonale were included in Group A, rest 10 patients of COPD with cor pulmonale were included in Group B.

\section{Echocardiography was performed in all patients according to the same Protocol Conventional Echocardiography}

Measurement of LV EF\% by M-mode by measurement of LV systolic and diastolic dimensions obtained through long-axis parasternal approach in M-mode projection. LV diastolic filling patterns were determined by the mitral inflow pulsed wave Doppler examination. In the apical 4-chamber view, the Doppler sample volume was placed in the middle of LV inflow tract $1 \mathrm{~cm}$ below the plane of mitral annulus between the mitral leaflet tips, where maximal flow velocity in early and late diastole was recorded.

The Right Ventricular Systolic Pressure (RVSP) was obtained from the velocity of tricuspid regurgitation. The value of $10 \mathrm{mmHg}$, obtained from the modified Bernoulli equation was added to the pressure gradient between the right ventricle and right atrium.

\section{Pulsed Wave Doppler Tissue Imaging (PW-DTI)}

PW-DTI was performed in apical 4 and 2 chamber views; the Doppler sample volume was placed at four different sites of the mitral annulus and major velocity time intervals recorded- IVCT (isovolumetric contraction time), IVRT (isovolumetric relaxation time) and $S$ wave duration (ejection time).

The regional myocardial velocity waves were systolic velocity ( $S$ wave; $\mathrm{cm} / \mathrm{s}$ ), peak early diastolic filling velocity $\left(E_{\mathrm{m}} ; \mathrm{cm} / \mathrm{s}\right)$ and peak late diastolic filling velocity $\left(A_{\mathrm{m}} ; \mathrm{cm} / \mathrm{s}\right)$, also $E_{\mathrm{m}} / A_{\mathrm{m}}$ ratio and $E_{\text {flow }} / E_{\mathrm{m}}$ were calculated.

\section{RESULTS}

\section{Statistical Analysis}

For statistical analysis data were entered into a Microsoft Excel spreadsheet and then analysed by SPSS 20.0.1 and Graph Pad Prism version 5. Data had been summarised as mean and standard deviation for numerical variables and count and percentages for categorical variables. Two-sample $\mathrm{t}$-tests for a difference in mean involved independent samples or unpaired samples. Unpaired proportions were compared by Chi-square test or Fisher's exact test, as appropriate. Z-test (Standard Normal Deviate) was used to test the significant difference of proportions. p- value $\leq 0.05$ was considered for statistical significance.

\begin{tabular}{|c|c|c|c|}
\hline & $\begin{array}{c}\text { No. of Total } \\
\text { Patients }\end{array}$ & $\begin{array}{c}\text { Percentage } \\
\text { (\%) }\end{array}$ & P-value \\
\hline $\begin{array}{c}\text { COPD without } \\
\text { Cor pulmonale }\end{array}$ & 42 & $80.7 \%$ & \multirow{2}{*}{$<0.0001$} \\
\cline { 1 - 4 } $\begin{array}{c}\text { COPD with Cor } \\
\text { pulmonale }\end{array}$ & 10 & $19.3 \%$ & \\
\hline \multicolumn{3}{|c|}{ Table 1. Incidence of Cor Pulmonale with COPD } \\
\hline
\end{tabular}

Out of the 52 COPD cases, $42(80 \%)$ were without cor pulmonale and $10(19.3 \%)$ were COPD with cor pulmonale

\begin{tabular}{|c|c|c|c|c|}
\hline Group & Male & Percentage (\%) & Female & Percentage (\%) \\
\hline A & 36 & $69.2 \%$ & 6 & $11.5 \%$ \\
\hline B & 8 & $15.3 \%$ & 2 & $3.9 \%$ \\
\hline \multicolumn{5}{|c|}{ Table 2. Sex Distribution } \\
\hline
\end{tabular}

$P=0.6415$

From the above table, it is observed that number of males in Group A and Group B were 36 (69.2\%) and 8 (15.3\%) respectively. Number of female in Group A and Group B were $6(11.5 \%)$ and $2(3.9 \%)$, respectively. Total number of male and female were 44 and 8 respectively (84.5\%) and (15.4\%) respectively with male: female ratio $5.5: 1$. 


\begin{tabular}{|c|c|c|}
\hline Name of the Disease & Smoking Association & Percentage \\
\hline COPD without Cor pulmonale (Group A, $\mathrm{n}=42$ ) & 30 & $71 \%$ \\
\hline COPD with Cor pulmonale (Group B, $\mathrm{n}=10$ ) & 10 & $100 \%$ \\
\hline
\end{tabular}

$\mathrm{p}=0.0536$.

From the above table, it has been observed that in Group A 30 (71\%) patients were smokers. In Group B, 100\% patients were smokers.

\begin{tabular}{|l|c|c|c|c|c|}
\hline & Group A (n = 42) & Percentage & Group B (n = 10) & Percentage & P-value \\
\hline 1. Dyspnoea & 42 & $100 \%$ & 10 & $100 \%$ & $<0.0001$ \\
\hline 2. Cough & 42 & $100 \%$ & 9 & $90 \%$ & 0.03846 \\
\hline 3. Sputum & 36 & $70 \%$ & 4 & $40 \%$ & 0.00208 \\
\hline 4. Wheeze & 20 & $38.4 \%$ & 4 & $40 \%$ & 0.6672 \\
\hline 5. Fever & 18 & $34.6 \%$ & 3 & $30 \%$ & 0.4593 \\
\hline 6. Haemoptysis & 2 & $3.8 \%$ & - & - & 0.48392 \\
\hline 7. PND & - & - & 1 & $10 \%$ & 0.03846 \\
\hline 8. Palpitation & - & - & $10 \%$ & 0.03846 \\
\hline \multicolumn{7}{|r|}{} \\
\hline
\end{tabular}

The most common presenting feature, both Group A and Group B were dyspnoea and cough, which were present in almost $100 \%$ of cases. Other presenting feature were productive mucoid sputum and wheeze. Haemoptysis and palpitation were only in 2 cases of COPD and 1 case of cor pulmonale respectively.

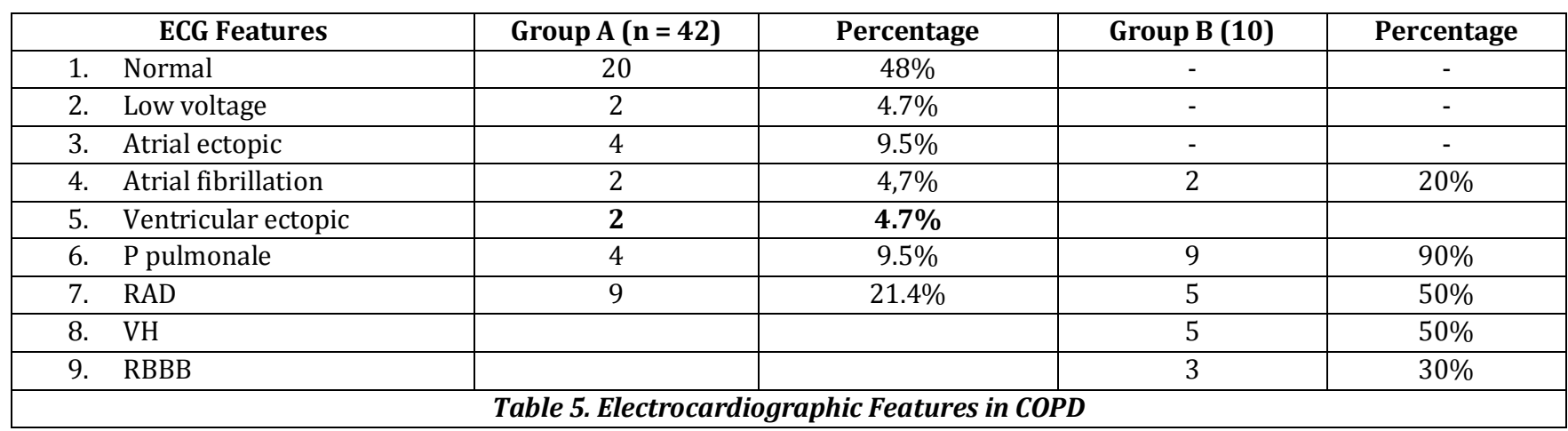

$\mathrm{p}=0.0151$

Above table showed that majority of patients in Group A had ECG within normal limit; 4 patients had atrial ectopic and 2 had ventricular ectopic. In Group B, P pulmonale was present in $9(90 \%)$ patients, right axis deviation and right ventricular hypertrophy in $5(50 \%)$ patients. In Group B, incomplete right bundle branch block was present in $3(30 \%)$ patients.

\begin{tabular}{|c|c|c|c|c|}
\hline Type of Disease & No. of Patients & Abnormal EF & Abnormal E/A Ratio & $\begin{array}{c}\text { Abnormal Fractional } \\
\text { Shortening }\end{array}$ \\
\hline COPD without cor pulmonale & 42 & $3(7.4 \%)$ & $13(31 \%)$ & $3(7.4 \%)$ \\
\hline COPD with cor pulmonale & 10 & $3(30 \%)$ & $10(100 \%$ & $3(30 \%)$ \\
\hline P-value & 0.04236 & $\mathrm{P}<0.0001$ & 0.04236 \\
\hline \multicolumn{2}{|r|}{ Table 6. Comparison of Incidence of Abnormal Systolic and Diastolic Function in COPD Patients } \\
\hline
\end{tabular}

Incidence of both systolic and diastolic dysfunction were higher in cor pulmonale patients in comparison to COPD without cor pulmonale patients.

\begin{tabular}{|c|c|c|c|}
\hline No. of Cases & RV Diameter (> 30 mm) & RV Wall Thickness (> 6 mm) & TR Jet \\
\hline COPD without cor pulmonale $(\mathrm{n}=42)$ & - & - & - \\
\hline COPD with cor pulmonale $(\mathrm{n}=10)$ & $6(60 \%)$ & $4(40 \%)$ & $4(40 \%)$ \\
\hline \multicolumn{2}{|r|}{ Table 7. Right Ventricular Wall Thickness and Diameter } \\
\hline
\end{tabular}

From the above table, it is seen that increased right ventricular diameter and wall thickness was present in patients with cor pulmonale. Right ventricular diameter was increased in $60 \%$ cases and wall thickness was increased in $40 \%$ cases. Pulse Doppler of Tricuspid valve showed TR jet in $40 \%$ cases of cor pulmonale. 


\begin{tabular}{|c|c|c|}
\hline & $\begin{array}{c}\text { COPD without } \\
\text { Cor pulmonale }\end{array}$ & $\begin{array}{c}\text { COPD Cor } \\
\text { pulmonale }\end{array}$ \\
\hline $\begin{array}{c}E \text { wave peak velocity by } \\
\text { mitral flow }\left(E_{\text {flow }}(\mathrm{m} / \mathrm{s})\right.\end{array}$ & $0.55 \pm 0.12$ & $0.54 \pm 0.12$ \\
\hline$E / A$ by flow (mean \pm SD) & $0.55 \pm 0.12$ & $0.54 \pm 0.12$ \\
\hline $\begin{array}{c}\text { E wave peak velocity by DTI } \\
\left(E_{\mathrm{m}}\right)(\mathrm{cm} / \mathrm{s})(\text { mean } \pm \text { SD) }\end{array}$ & $7.08 \pm 1.26$ & $8.22 \pm 1.72$ \\
\hline$E_{\mathrm{m}} / A_{\mathrm{m}}$ by DTI (mean \pm SD) & $0.64 \pm 0.06$ & $0.76 \pm 0.24$ \\
\hline IVRT (msec.) (mean \pm SD) & $72.32 \pm 8.46$ & $73.22 \pm 6.28$ \\
\hline \multicolumn{2}{|c|}{ Table 8. Left Ventricular Diastolic Function }
\end{tabular}

From the above table, left ventricular diastolic function assessment revealed a significant difference in $\mathrm{E}$ wave peak velocity and $E / A$ ratio. Isovolumetric Relaxation Time (IVRT) was not significantly different.

\section{DISCUSSION}

Cardiovascular manifestation is a substantial cause of morbidity in COPD patients. Pulmonary arterial hypertension is responsible for this cardiac manifestation in most COPD patients. Though in COPD right ventricle get maximum brunt, which is very obvious by its clinical presentation, left ventricular dysfunction occurs simultaneously which may not be obvious by its clinical manifestation. In absence of clinically recognisable left ventricular dysfunction noninvasive evaluation by Echocardiography can establish this dysfunction in early stages before the development of overt failure. This present study was designed to find out left ventricular dysfunction by Echocardiography (M. Mode, 2-D and Doppler) in COPD with and without cor pulmonale.

For this, 52 patients of COPD who were admitted to the Department of General Medicine, Cardiology and Chest, to Chest Department and Cardiology at SSKM Hospital and IPGMER Kolkata during the period from January 2015 to December 2016 were taken under study. Out of these, 42 patients were without cor pulmonale and 10 patients were with cor pulmonale. Hypertension, coronary heart disease or vascular heart disease were excluded from the study.

The male: female ratio was found to be 5.5: 1 in this present study. In our country, Viswanathan et al (1977) found male: female ratio to be 5: 1 in Delhi. Worker like Gocke and Webston (1985) have noted the ratio to be 10: 1 and $8: 1$, respectively.

The commonest age incidence was observed in this study between 56 - 65 years. Higgins ${ }^{6}$ (1982) observed that the incidence of COPD increase with age and higher in men than in women, particularly above 45 years of age. S. Vilkman and Colleagues (1996) reported that in man the need of hospital treatment for COPD started to rise sharply after the age of 50 .

Jacob (1980), Higgins et al (1982), Crofton and Douglas (2000) are of opinion that COPD is more prevalent in lower socio-economic groups. Probably, it is due to untreated or inadequately treated respiratory infections in addition to other risk factors like environmental condition, etc. In the present study, $63.4 \%$ (vide Table 6) of cases came from lower socio-economic groups, such observation was also confirmatory with Covaraugh. Fraser and Pare (1986), Bartolome R. Coil (1998) also mentioned that poverty is the possible risk factor for COPD.

As regards to the association of smoking with COPD, association of smoking is observed in majority of cases $(78 \%)$, which is well compared with the observation of various worker (Bartolen R. Coil, 1998). In our country, Mallick (1987) and Mallick et al (1988) observed smoking habit in $78 \%$ and $71 \%$ of COPD patients, respectively. In this study, it was found that 12 out of 13 bronchial asthma patients were non-smoker. Rest of all patients were smoker.

Duration of illness prior to the first attendance varies between 45 days to more than 10 years. Majority being seen between 3 years to 10 years. The asthmatic group usually presented within 1 year of their illness. These findings are in agreement with other workers. Viswanathan et al (1977) had also made similar observation. But in cases of cor pulmonale, $60 \%$ of patients presented 10 years after their first symptoms.

Dyspnoea was the most common symptom in patients with COPD, which was present in $100 \%$ of cases in both groups, COPD with or without cor pulmonale. As regards to symptoms, the present study corroborates with the findings of Crofton and Douglas (2000) and Tisi (1980). Other presenting symptoms were cough, sputum and wheezing, and fever. Haemoptysis were present in few cases (3.8\%). Similar patterns of symptoms were observed by Salvato (1978).

With regards to physical sign vide Table 9, the present study revealed that all had one or the other abnormality in the chest. Crofton and Douglas (2000) reported that wheeze is usually audible all over the chest in almost all cases of asthma in a severe attack and expiratory rhonchi are usually heard during the mild attack. In the present series, all the asthmatic had rhonchi during admission. Bohadma (1988) found that most common physical finding in chronic bronchitis is the presence of rhonchi, mostly expiratory, but sometimes inspiratory as well and there may be crepitation especially at bases in presence of acute exacerbation. In the present study, all the patients were hospitalised, had one or more physical signs and observations are well corroborated with the observation of the above cited workers.

Fraser et al (1986) found $21 \%$ of the chronic bronchitis had normal chest $x$-ray, in contrast to the $10 \%$ of the present study. It may be due to the fact that all the chronic bronchitis patients had same or other complication. They found increased broncho-vascular marking, tubular heart shadow and low and flat diaphragm in 58\%, 13\% and 68\% cases respectively. In present study, the corresponding findings are $47 \%, 1.4 \%$ and $52 \%$ respectively. In emphysematous patient in this study, the findings such as low flat diaphragm, increased lucency and tubular heart are broadly in agreement with the observation of other workers.

In asthmatic group of the present study, half of them are radiologically normal and rest half shows increased lucency and low flat diaphragm. Fraser and Pare (1986) observed $56 \%$ of asthmatic had normal chest x-ray and rest $44 \%$ had over inflation and increased lucency with hilar prominence. The finding of the present study is more or less consistent with them. Broadly speaking the radiological findings in the present study are in agreement with that of above cited workers.

David Camper et al (1975), found in a series of 103 patients of COPD that $45.6 \%$ of his patients met one or more electrocardiographic changes of COPD. M.L. Murphy et al (1974) in a study of 71 patients in whom EGG changes during life were correlated with right ventricular mass at autopsy, found that 30 had definite and 3 had probable right ventricular hypertrophy, 20 had normal ventricular weight 
and 18 had left ventricular hypertrophy. Three criteria were found to be most reliable indicator of RVH: right axis deviation $\left(>110^{\circ}\right)$; S1, S2, S3 pattern and an RS ratio in V5 (< 1.0).

F. J. C. Millard and Colleagues (1967) reported that 20 of his 46 COPD patients had isolated right ventricular hypertrophy; 16 of these had right axis deviation $\left(+90^{\circ}\right.$ to $+180^{\circ}$ ). Among the 20 patients with isolated right ventricular hypertrophy, 14 had an S wave of $10 \mathrm{~mm}$ or more in VI. Only 8 of these 20 had "P" pulmonale.

In this present study, ECG was normal in 20 patients (48.5\%). In Group A, 9 (21.9\%) patients showed evidence of right axis deviation. 2 patients (4.7\%) had low voltage, 4 (9.5\%) had atrial ectopic, 2 (4.7\%) had ventricular ectopic.

In Group B, "P" pulmonale was present in 9 (90\%) patients, right ventricular hypertrophy and right axis deviation in 5 patients (50\%). $2(20 \%)$ patients showed evidence of atrial fibrillation. Incomplete RBBB was present in $3(30 \%)$ patients and $\mathrm{R} / \mathrm{S}<1$ in V5 was present in $4(40 \%)$ patients.

All above patients were studied by Echocardiogram (Mmode, 7; D, Doppler). Ejection fraction, fractional myocardial shortening were calculated. Left ventricular inflow Doppler study was done. E/A ratio (Ratio of peak velocity of early diastolic filling and peak velocity of late diastolic filling) was calculated 3 of the 10 patients (30\%) with cor pulmonale had reduced ejection fraction. 3 of the patients in Group A (7.14\%) without cor pulmonale had reduced ejection fraction. Similarly, fractional myocardial shortening was reduced in $3(30 \%)$ patients with cor pulmonale and 3 patients without cor pulmonale (7.14\%).

Settle and Colleagues (1975) measured left ventricular ejection fraction in 120 patients of COPD using radionuclide ventriculography and found 32 patients who had abnormal left-sided ejection fraction, whereas value was less than $40 \%$ in only $19(21 \%)$ of these patients. Of these 12 had autopsy and clinical evidence of co-existence coronary artery disease. Subsequently, Kline and Colleagues (1977) using first pass radionuclide technique reported similar finding. They found 2 of 20 patients with COPD had abnormal left ventricular ejection fraction. In the series by Mathay and Colleagues (1980) only 4 of 30 patients (13\%) had resting abnormalities in left ventricular ejection fraction, but all of them had assessed left ventricular function by invasive method like radionuclide angiography. M.L Render and Colleagues ${ }^{7}$ also showed 25 of his 77 patients (32\%) with COPD had left ventricular dysfunction (LVEF $<40 \%$ ) associated with wall motion abnormalities on radionuclide ventriculogram.

Vizza CD and Colleagues (1998) showed in a study of 434 COPD patients by using echocardiography technique that left ventricular dysfunction (LVEF $<45 \%$ ) was present only in $6.4 \%$, but it was common in the group of pulmonary hypertension (19.6\%). This finding was corroborative with the result of our study.

Along with systolic functional abnormality, in COPD patients with or without cor pulmonale have abnormalities in diastolic function due to reduced compliance of left ventricle (Feigenbaum $\mathrm{H}$ 1994). Isovolumetric relaxation period is prolonged, mitral valve opening is significantly delayed in relation to aortic valve closure. Similarly, early diastolic filling height decreases, but late diastolic filling height due to atrial contraction increases. Hence, the ratio of early diastolic filling height (E) and late diastolic filling height (A) decreases.

Left ventricular inflow study was done. Both early diastolic filling height (E) and late diastolic filling height was determined, E/A ratio was calculated. It was observed that there were reduction of height of $\mathrm{E}$ wave, increase in the height of A wave and alteration of E/A ratio in both groups of COPD with or without cor pulmonale.

In the present work, left ventricular systolic function did not significantly differ between different COPD with or without cor pulmonale, while left ventricular diastolic function assessment revealed a significant difference in $\mathrm{E}$ wave peak velocity and $E / A$ ratio (both measured by DTI echocardiography) COPD patients.

All $10(100 \%)$ patients with cor pulmonale and 13 (31\%) of the 42 patients without cor pulmonale had reduced $\mathrm{E}$ and also E/A ratio.

In this study, systolic as well as diastolic dysfunction of left ventricle were noted in both the patients of COPD with or without cor pulmonale and it was observed that the incidence of left ventricular diastolic dysfunction was higher than left ventricular systolic dysfunction. Left ventricular diastolic and systolic function deteriorates with gradual development of pulmonary arterial hypertension and subsequently right ventricular dilation and/or hypertrophy.

This study showed that left ventricular dysfunction occurs in patients with COPD and that is significantly more in COPD patients associated with cor pulmonale. Moreover, of diastolic dysfunction in both groups of COPD and cor pulmonale was higher than systolic dysfunction.

\section{CONCLUSION}

The present study was undertaken to evaluate systolic and diastolic dysfunction of left ventricle, if any, in COPD patients noninvasively with Echocardiography. Non-invasive evaluation of left ventricular function by Echocardiography was found to be helpful in detecting left ventricular dysfunction in patient of COPD with or without cor pulmonale. The present study showed incidence of left ventricular diastolic function abnormality was more than the incidence of systolic function abnormality and it was observed that cor pulmonale patients had more incidence of both diastolic and systolic dysfunction than patients of COPD without cor pulmonale. COPD patients with pulmonary hypertension are more liable to LV diastolic and global dysfunction than normal pulmonary pressure COPD patients. Doppler tissue echocardiography is a better tool in the assessment of left ventricular function. Therefore, it can be concluded that systolic and diastolic dysfunction can be present without being overt clinical manifestation of left ventricular dysfunction.

\section{REFERENCES}

[1] Lopez AD, Shibuya K, Rao C, et al. Chronic obstructive pulmonary disease: current burden and future projections. Eur Respir J 2006;27(2):397-412.

[2] Barnes PJ, Celli BR. Systemic manifestations and comorbidities of COPD. Eur Respir J 2009;33(5):116585. 
[3] Barberà JA, Blanco I. Pulmonary hypertension in patients with chronic obstructive pulmonary disease: advances in pathophysiology and management. Drugs 2009;69(9):1153-71.

[4] MacNee W. Pathophysiology of cor pulmonale in chronic obstructive pulmonary disease. Am J Respir Crit Care Med 1994;150(4):1158-68.

[5] Higgins MW, Keller JB, Landis JR, et al. Risk of chronic obstructive pulmonary disease. Collaborative assessment of the validity of the Tecumseh index of risk. Am Rev Resp Disease 1984;130(3):380-5.
[6] Render ML, Weinstein AS, Blaustein AS. Left ventricular dysfunction in deteriorating patients with chronic obstructive pulmonary disease. Chest 1995;107(1):162-8.

[7] Lee-Chiong TL, Matthay RA. Pulmonary hypertension and cor pulmonale in COPD. Semin Respir Crit Care Med 2003;24(3). 